\title{
Sintomas de Depressão, Ansiedade e Estresse em Usuários de Drogas em Tratamento em Comunidades Terapêuticas
}

\author{
Ilana Andretta ${ }^{1}$ \\ Jéssica Limberger ${ }^{1}$ \\ Jaluza Aimèe Schneider ${ }^{2}$ \\ Luana Thereza Nesi de Mello' \\ ${ }^{1}$ Universidade do Vale do Rio dos Sinos, São Leopoldo, RS \\ ${ }^{2}$ Pontifícia Universidade Católica do Rio Grande do Sul, Porto Alegre, RS
}

\begin{abstract}
Resumo
Sintomas de depressão, ansiedade e estresse podem interferir negativamente no progresso do tratamento de usuários de substâncias. Este estudo objetivou avaliar os níveis de depressão, ansiedade e estresse em homens e mulheres em tratamento em Comunidades Terapêuticas, relacionando tais níveis com o tipo de substância utilizada e dados sociodemográficos. Utilizou-se um Questionário de Dados Sociodemográficos e de Uso de Drogas e a escala DASS-21. Participaram 115 homens e 53 mulheres, em tratamento, totalizando 168 indivíduos, com idade média de 32 anos $(D P=8,7)$. A maioria eram usuários de tabaco e crack, este último associado às mulheres. Identificou-se que os usuários de tabaco e os usuários de drogas que não tinham emprego se associaram a níveis mais altos dos sintomas de depressão, ansiedade e estresse. Conclui-se que tais estratégias podem auxiliar na diminuição dos sintomas avaliados pelo DASS-21, melhorando as condições de vida para o enfrentamento do transtorno por uso de substâncias.

Palavras-chave: depressão, ansiedade, stress, drogas (uso)
\end{abstract}

Symptoms of Depression, Anxiety and Stress in Drug Users undergoing Treatment in Therapeutic Communities

\section{Abstract}

Symptoms of depression, anxiety and stress may interfere negatively in the progress of treatment of substance abusers. This study aimed to evaluate the levels of depression, anxiety and stress in men and women undergoing treatment for substance use disorder in therapeutic communities, relating such levels with the type of substance used and sociodemographic data. We used a Sociodemographic and Drug Use Data Questionnaire and the DASS-21. Participants included 115 men and 53 women on treatment $(M=32$ years; $S D=8.7)$. Most were users of tobacco and crack, the latter associated with women. Tobacco and other drug users who were unemployed were associated with higher levels of depression, anxiety, and stress symptoms in the DASS-21. The results do not allow a causal relationship between the variables, but highlight the relevance of including in the treatments, strategies for smoking cessation and professional reintegration. Such strategies may help to reduce the symptoms evaluated by the DASS-21, improving living conditions for coping with substance use disorders.

Keywords: Depression; Anxiety; Stress; Drug use.

\section{Síntomas de Depresión, Ansiedad y Estrés en Usuarios de Drogas en Tratamiento en Comunidades Terapéuticas}

\section{Resumen}

Los síntomas de depresión, ansiedad y estrés pueden afectar de forma negativa el progreso del tratamiento de usuarios de drogas. El objetivo de este estudio fue evaluar los niveles de estos síntomas en los hombres y las mujeres en tratamiento en Comunidades Terapéuticas, relacionando tales niveles con el tipo de sustancia utilizada y datos sociodemográficos. Se utilizó un Cuestionario sobre Datos Sociodemográficos y Uso de Drogas y escala DASS-21. Participaron 115 hombres y 53 mujeres en tratamiento, con edad media de 32 años, DP $=8,7)$. La mayoría eran consumidores de tabaco y crack, este último asociado a las mujeres. Los usuarios de drogas y tabaco que no tenían empleo, se asociaron a niveles más altos de síntomas de depresión, ansiedad y estrés. Se concluye que tales estrategias pueden auxiliar en la disminución de los síntomas evaluados por la DASS-21, mejorando las condiciones de vida para el enfrentamiento de los trastornos por uso de sustancias.

Palabras-clave: Depresión; Ansiedad; Estrés; Drogas (uso)

\section{Introdução}

Mundialmente, o uso de drogas caracteriza-se como um grave problema social e de saúde pública, causando danos consideráveis ao indivíduo, além de desafios no sistema de controle da violência advinda do tráfico e da rápida evolução de novas substâncias psicoativas (United Nations Office on Drugsand Crime
[UNODC], 2013). Dados do Relatório Mundial sobre o uso de drogas apontam que em 2012, entre 162 e 324 milhões de pessoas (de 3,5\% a 7,0\% da população), com idade entre 15 e 64 anos, consumiram ao menos uma vez algum tipo de droga ilícita. Também se destaca as consequências individuais que o uso de drogas envolve, considerando maior vulnerabilidade a doenças físicas, prejuízos nas relações familiares, sociais 
e profissionais, além de maior incidência de psicopatologias (Diehl, Cordeiro, \& Laranjeira, 2011; Fox, Oliver, \& Ellis, 2013).

Diversos estudos discutem o alto índice de comorbidades psiquiátricas em indivíduos que usam substâncias, destacando-se os transtornos depressivos e de ansiedade (Duailibi, Ribeiro, \& Laranjeira, 2008; Hess, Almeida, \& Moraes, 2012; Scheffer, Passa, \& Almeida, 2010). Na Austrália, um estudo identificou que $76 \%(n=72)$ dos participantes com transtorno por uso de substâncias apresentaram sintomas de transtorno depressivo e 71\% ( $n=67)$ de transtorno de ansiedade (Staiger, Thomas, Ricciardelli, \& McCabe, 2011). Um estudo holandês, do Centro de Estudo de Depressão e Ansiedade do país, teve como intuito avaliar se o uso de álcool está relacionado a persistência de sintomas de ansiedade e depressão (Boschloo et al., 2012). Participaram da pesquisa 1.369 adultos, com diagnóstico de transtorno depressivo e de ansiedade. Os resultados identificaram que aqueles que possuíam uma dependência grave de álcool foram relacionados a persistência do curso dos sintomas de ansiedade e depressão por até dois anos, porém o abuso de álcool não apresentou relação com tais sintomas (Boschloo et al., 2012).

Os sentimentos negativos sobre si, a redução de satisfação nas atividades diárias e o humor deprimido afetam diretamente a qualidade de vida do indivíduo (Beck \& Alfrod, 2011; Castro et al., 2010). Dessa forma, pacientes em tratamento para transtorno por uso de substâncias com sintomas depressivos, possuem menor qualidade de vida e maiores índices de retorno ao uso da droga em comparação a pacientes que não possuem tal sintomatologia (Saatcioglu, Yapici, \& Cakmak, 2008). Em um estudo brasileiro realizado em um Centro de Atendimento Psicossocial Álcool e Drogas (CAPSad), a presença de sintomas depressivos correlacionou-se negativamente com a qualidade de vida de usuários de substâncias, prejudicando a fase de recuperação dos pacientes estudados (Marcon, Xavier, Barcelon, Espinosa, \& Barbosa, 2014).

Percebe-se a tendência de maiores prejuízos do uso de substâncias naqueles usuários que apresentam, de forma concomitante, a depressão relação que já foi confirmada em usuários de substâncias injetáveis (Conner, Pinquart, \& Holbrook, 2008). Nessa perspectiva, justifica-se o fato de que sintomatologia depressiva implica em uma maior probabilidade de internação hospitalar para tratamento decorrente do uso de drogas e no aumento da busca de tratamento ambulatorial por essa população (Argimon et al., 2013).
Além da depressão, os sintomas de ansiedade também têm se destacado na discussão sobre consumo de drogas, identificando-se possíveis relações com os transtornos por uso de substâncias (Marmorstein, 2012). Segundo o DSM-5, a ansiedade trata-se da antecipação de ameaça, que ao ocorrer de forma desadaptativa pode ser caracterizada como um transtorno psicopatológico de ansiedade (American Psychiatric Association [APA], 2014). Em um estudo americano, identificou-se a maior probabilidade de transtornos por uso de substâncias em indivíduos com algum transtorno de ansiedade internalizante, sendo que o transtorno de estresse pós-traumático apresentou maiores índices entre eles (Hofmann, Richey, Kashdan, \& McKnight, 2009).

Tratando-se de estudos nacionais, Hess et al. (2012) encontraram associação positiva entre transtorno de ansiedade generalizada e transtorno por uso de substâncias em indivíduos em tratamento de internação. Em uma amostra de adolescentes, a ansiedade moderada foi relacionada com o uso frequente do álcool e, também, apresentou maior incidência naqueles que já haviam consumido solvente, energético e/ou cigarro (Lopes \& Rezende, 2013). Em 84 pacientes de um ambulatório de psiquiatria, identificou-se uma frequência de $75 \%$ para transtornos de ansiedade, no qual 21,4\% da amostra eram usuários de tabaco (Munaretti \& Terra, 2007). Também foram identificados maiores níveis de ansiedade em usuários de cocaína, cracke e álcool quando comparados a não usuários de substâncias (Scheffer et al., 2010).

Uma terceira variável importante a ser analisada em relação ao transtorno por uso de substâncias trata-se do estresse. Eventos estressores ao longo da vida do indivíduo podem contribuir para o uso de substâncias, apresentando-se como uma maneira desadaptativa de aliviar tais sintomas (Enoch, 2003). Além disso, altos níveis de eventos estressantes entre usuários de drogas em tratamento e em abstinência podem dificultar o manejo de enfrentamento de suas angústias e aumentar o desejo pela substância, elevando as possibilidades de retorno ao uso de drogas (Sinha, 2009). Goeders (2003) ressalta a importância da diminuição do estresse como um fator de proteção, possibilitando a redução dos desejos de utilizar drogas e o auxílio da promoção da abstinência em usuários que estão em tratamentos.

Diante desse contexto, ressalta-se a relevância de considerar os níveis de depressão, ansiedade e estresse durante o tratamento de usuários de drogas, a fim de escolher e direcionar as intervenções de acordo com as demandas encontradas e diminuir possíveis riscos 
de retorno para o uso de drogas. Porém, destaca-se a necessidade de conhecer quais drogas estão relacionadas com tais sintomatologias, além de investigar características sociodemográficas que possam também estar associadas. Neste sentido, objetiva-se avaliar os níveis de depressão, ansiedade e estresse em homens e mulheres em tratamento para transtorno por uso de substâncias. Além disso, objetiva-se avaliar a relação dos níveis de depressão, ansiedade e estresse com o tipo de substância e com as características sociodemográficas como sexo, escolaridade, idade, estado conjugal, religião, nível socioeconômico e trabalho.

\section{Método}

\section{Participantes}

Foram avaliados para participar deste estudo 218 homens e mulheres em tratamento em 15 Comunidades Terapêuticas da região noroeste e metropolitana do estado do Rio Grande do Sul. Segundo a Federação Brasileira de Comunidades Terapêuticas (FEBRACT, 2016) há 97 Comunidades Terapêuticas filiadas no território nacional, destas 30 localizadas no estado da coleta de dados. A seleção dos locais de tratamento e dos participantes foi por conveniência, possuindo os seguintes critérios de inclusão: ter entre 18 e 59 anos, estar abstinente há pelo menos sete dias e possuir critérios diagnósticos para Transtorno por Uso de Substâncias, de acordo com o DSM-5 (APA, 2014). Foram excluídos participantes que não tivessem completado os instrumentos, com síndrome psicótica (verificada por meio da Mini International Neuropsychiatric Interview) (Amorim, 2000), com prejuízos cognitivos (verificados através do Screening Cognitivo do WAIS-III) (Wechsler, 1997), justificando-se por tais características dificultarem a compreensão e orientação para o preenchimento dos instrumentos. Ao final do processo de avaliação atenderam os critérios de inclusão deste estudo 168 participantes.

\section{Instrumentos}

O Questionário de Dados Sociodemográficos e Padrão de Uso de Drogas, desenvolvido pelo grupo de pesquisa tem como objetivo traçar um perfil dos usuários. É composto por dados sociodemográficos, dados de classificação socioeconômica de acordo com os Critérios de Classificação Econômica Brasil (CCEB) da Associação Brasileira de Empresas de Pesquisas (ABEP, 2015), dados familiares, padrão de uso de drogas (tipo e tempo de uso de cada droga utilizada) e itens do DSM-5 (APA, 2014) para diagnóstico de transtorno por uso de substâncias.

A Depression, Anxiety and Stress Scale (DASS-21), versão curta de 21 itens e que possui três subescalas, do tipo Likert, de 4 pontos. Cada subescala é composta por sete itens, que avaliam simultaneamente os estados emocionais de depressão, ansiedade e estresse (Apóstolo, Mendes, \& Azeredo, 2006). Os sintomas avaliados na subescala de depressão se referem a inércia, anedonia, disforia, falta de interesse/envolvimento, auto-depreciação, desvalorização da vida e desânimo. $\mathrm{Na}$ subescala de ansiedade são avaliados sintomas de excitação do sistema nervoso autônomo; efeitos musculoesqueléticos; ansiedade situacional; experiências subjetivas de ansiedade. As questões da subescala de estresse avaliam os sintomas de dificuldade em relaxar; excitação nervosa; fácil perturbação/agitação; irritabilidade/reação exagerada e impaciência. A DASS-21 é validada para a língua portuguesa por Apóstolo e colaboradores (2006), possuindo confiabilidade adequada para cada uma das subescalas: depressão $(\alpha=$ $0,9)$, ansiedade $(\alpha=0,86)$ e estresse $(\alpha=0,88)$. Trata-se de uma escala autoaplicável, realizada diante da explicação do enunciado, que indica ao participante optar pelo nível da sintomatologia descrita na afirmação, pontuando de zero à três pontos, em relação a sua aplicabilidade na última semana. Obtém-se o resultado através do somatório das respostas aos itens que compõem cada uma das três subescalas, podendo variar em cada uma de zero a 21 pontos, sendo que o resultado mais alto indica a sintomatologia mais elevada relacionada aos estados emocionais. A partir da pontuação, a classificação corresponde a normal, médio, moderado, severo e extremamente severo (Apóstolo et al., 2006).

\section{Procedimentos}

Este estudo faz parte de um projeto maior que obteve aprovação do Comitê de Ética em Pesquisa Comitê de Ética em Pesquisa da Unisinos, sob o parecer $\mathrm{n}^{\circ} 13.172$. Para a coleta de dados, em um primeiro momento, foram realizados contatos com 15 locais e tratamento de internação para usuários de drogas, a fim de obter a permissão dos responsáveis para a realização da coleta de dados em local cedido na própria instituição. Após, foram realizados convites a todos os indivíduos que estavam internados nas comunidades no período de coleta de dados para participar do estudo com a explicação sobre os objetivos, assim como fornecimento de informação sobre anonimato, possíveis benefícios, autonomia de se desvincular a qualquer 
momento, entre outras. Ao aceitarem participar da pesquisa, de forma voluntária, foi assinado o Termo de Consentimento Livre e Esclarecido (TCLE) em duas vias, ficando uma via com o participante e outra com o pesquisador responsável. Em seguida, foi iniciada a avaliação através da aplicação dos instrumentos descritos, de forma individual, em um local disponibilizado pela instituição de tratamento. A coleta de dados teve uma duração de seis meses e foi realizada por integrantes do grupo de pesquisa (estudantes de graduação e mestrandos em psicologia) previamente treinados.

\section{Análise de Dados}

Os dados foram analisados pelo Statistical Package for Social Sciences (SPSS), versão 20.0. Foram realizadas análises descritivas dos dados obtidos no Questionário de dados sociodemográficos e padrão de uso de drogas e dos itens da DASS-21, contemplando porcentagem, média e desvio-padrão da amostra. A fim de avaliar as diferenças significativas entre grupos, em relação às variáveis contínuas, foram estimadas as médias e desvios-padrão, aplicando o Teste t de Student Independente, sendo a diferença considerada significativa se a probabilidade do erro tipo I (a) tivesse valor $\leq 5 \%$ ( $p \leq$ $0,05)$. Foram utilizados testes qui-quadrado (teste exato de Fisher) para avaliar as associações entre as variáveis categóricas sobre aspectos sociodemográficos, padrão de consumo de substância e níveis de sintomatologia avaliada pelo DASS-21. Para maior poder estatístico, foram agrupados os resultados da DASS-21 em severo e extremamente severo, moderado, e normal e médio em função dos prejuízos dos escores nos participantes. Por fim, para fins de avaliação da confiabilidade do instrumento, aplicou-se o coeficiente do alfa de Cronbach (Sampieri, Collado, \& Lucio, 2013).

\section{Resultados}

A amostra foi de 168 participantes, sendo 68,5\% homens $(n=115)$ e $31,5 \%$ mulheres $(n=53)$, com média de idade de 32 anos $(D P=8,7)$. O tempo médio de abstinência dos participantes foi de 128,8 dias (DP $=176,6)$. A maioria revelou ser praticante de alguma religião $70,3 \%(n=158)$. Houve predominância de indivíduos de etnia caucasiana com 74,4\% $(n=125)$, solteiros $63,7 \%(n=107)$ e que tinham ao menos um filho $67,9 \%(n=114)$. Em relação à escolaridade, $46,4 \%$ participantes $(n=78)$ tinham apenas ensino fundamental incompleto e a maioria trabalhava antes da internação $67,3 \%(n=113)$.
No que diz respeito ao uso de substâncias, o uso de crack foi predominante, sendo relatada por 92,3\% dos participantes $(n=155)$, seguida do tabaco relatado por $42,9 \%$ dos participantes $(n=72)$. As demais drogas de uso dos indivíduos da amostra foram: 38,1\% ( $n$ = 64) álcool; 36,9\% $(n=62)$ cocaína; 21,4\% $(n=36)$ maconha e 4,8\% $(n=8)$ solvente.

$\mathrm{Na}$ comparação do sexo em relação ao uso de determinados tipos de drogas (Tabela 1), ocorreu associação estatística significativa em relação ao crack $(p=$ $0,010)$ apontando que as mulheres apresentaram associação significativa ao consumo de crack, $34,2 \% \quad(n=$ 53). As demais drogas, quando comparadas ao sexo, não apresentaram associações significativas, apontando que, o uso de álcool, tabaco, maconha cocaína e solvente, independem do sexo. Quando a idade foi comparada ao uso e não uso de determinados tipos de drogas, as diferenças significativas não se configuraram, apontando que a média de idade independe do uso das drogas avaliadas neste estudo.

Para a utilização do DAAS-21, foi realizada a análise de confiabilidade pela medida de consistência interna através do coeficiente alfa de Cronbach. A partir dos três fatores apontados pela leitura original do instrumento, foram obtidas as estimativas referentes às medidas descritivas (média e desvio padrão), correlação item-total e coeficiente alfa de Cronbach e este mesmo coeficiente se item deletado sobre cada fator (Tabela 2).

De acordo com a avaliação pelo DASS-21, a maioria dos participantes apresentou classificação normal nos sintomas de depressão, ansiedade e estresse, conforme pode ser observado na Tabela 3. Não foram encontradas diferenças estatisticamente significativas entre as seguintes variáveis: sexo, escolaridade, idade, estado conjugal, religião e nível socioeconômico em relação aos níveis de ansiedade, depressão e estresse. Houve relação significativa entre os sintomas de depressão e estar trabalhando atualmente. Participantes que trabalhavam associaram-se $(p=0,005)$ a níveis normais, médios e moderados de depressão. Participantes que não trabalhavam associaram-se aos níveis normais e severos de depressão $(p=0,005)$. Os índices de sintomas de ansiedade também se associaram à variável estar trabalhando atualmente $(p=0,242)$. Índices normais de estresse associaram-se com estar trabalhando atualmente, enquanto índices extremamente severos relacionaram-se com não estar trabalhando.

No que diz respeito ao uso de drogas, houve uma associação significativa entre o uso de tabaco e níveis de severos e extremamente severos de depressão, ansiedade 
Tabela 1

Distribuição Absoluta e Relativa para o Sexo, e Média e Desvio Padrão segundo o Uso de Drogas

\begin{tabular}{|c|c|c|c|c|c|c|c|c|c|c|}
\hline \multirow{3}{*}{ Drogas } & \multirow{2}{*}{\multicolumn{2}{|c|}{$\begin{array}{l}\text { Total amostra } \\
\qquad(n=168)^{*}\end{array}$}} & \multicolumn{5}{|c|}{ Sexo } & \multicolumn{3}{|c|}{ Idade } \\
\hline & & & \multicolumn{2}{|c|}{$\begin{array}{l}\text { Masculino } \\
(n=115)\end{array}$} & \multicolumn{2}{|c|}{$\begin{array}{c}\text { Feminino } \\
(n=53)\end{array}$} & \multirow[t]{2}{*}{$\mathrm{p}$} & \multirow[t]{2}{*}{ Média } & \multirow{2}{*}{$\begin{array}{l}\text { Desvio } \\
\text { padrão }\end{array}$} & \multirow[t]{2}{*}{$\mathrm{p} ¥$} \\
\hline & $\mathrm{N}$ & $\%$ & $\mathrm{~N}$ & $\%$ & $\mathrm{n}$ & $\%$ & & & & \\
\hline \multicolumn{11}{|l|}{ Álcool } \\
\hline Sim & 64 & 38,1 & 47 & 40,9 & 17 & 32,1 & 0,275 | & 33,5 & 9,8 & 0,101 \\
\hline Não & 104 & 61,9 & 68 & 59,1 & 36 & 67,9 & & 31,1 & 7,9 & \\
\hline \multicolumn{11}{|l|}{ Tabaco } \\
\hline Sim & 72 & 42,9 & 44 & 38,3 & 28 & 52,8 & $0,076 \mathbb{1}$ & 31,8 & 8,7 & 0,727 \\
\hline Não & 96 & 57,1 & 71 & 61,7 & 25 & 47,2 & & 32,2 & 8,8 & \\
\hline \multicolumn{11}{|c|}{ Maconha } \\
\hline Sim & 36 & 21,4 & 25 & 21,7 & 11 & 20,8 & 1,000 | & 30,3 & 10,5 & 0,186 \\
\hline Não & 132 & 78,6 & 36 & 78,7 & 90 & 79,2 & & 32,5 & 8,1 & \\
\hline \multicolumn{11}{|l|}{ Cocaína } \\
\hline Sim & 62 & 36,9 & 47 & 40,9 & 15 & 28,3 & $0,117 \boldsymbol{q}$ & 31,0 & 9,1 & 0,254 \\
\hline Não & 106 & 63,1 & 68 & 59,1 & 38 & 71,7 & & 32,6 & 8,5 & \\
\hline \multicolumn{11}{|l|}{ Crack } \\
\hline Sim & 155 & 92,3 & 102 & 88,7 & 53 & 100 & $0,010 \$$ & 31,9 & 8,5 & 0,608 \\
\hline Não & 13 & 7,7 & 13 & 11,3 & 0 & 0 & & 33,2 & 10,9 & \\
\hline \multicolumn{11}{|l|}{ Solvente } \\
\hline Sim & 8 & 4,8 & 7 & 6,1 & 1 & 1,9 & $0,235 \$$ & 32,3 & 6,3 & 0,943 \\
\hline Não & 160 & 95,2 & 108 & 93,9 & 52 & 98,1 & & 32,0 & 8,8 & \\
\hline
\end{tabular}

Nota. $*$ Percentuais obtidos sobre o total da amostra. ¥: Teste $t$ Student para grupos independentes;

q: Teste Qui-Quadrado de Pearson com correção de continuidade;

ऽ: Teste Exato de Fisher.

e estresse. Além da subdivisão do instrumento (normal, médio, moderado, severo e extremamente severo), também foram realizadas análises a partir de três agrupamentos (1 - normal e médio; 2 - moderado; 3 - severo e extremamente severo), identificando que tais diferenças estatísticas se mantiveram, conforme pode ser observado na tabela 4 . Tratando-se das outras substâncias avaliadas (álcool, maconha, solvente, cocaína e crack) não foram encontradas associações significativas em relação aos sintomas de depressão, ansiedade e estresse avaliados pelo DASS-21.

\section{Discussão}

Este estudo contemplou seu objetivo proposto, de avaliar os níveis de depressão, ansiedade e estresse em homens e mulheres em tratamento para transtorno por uso de substâncias, relacionando tais níveis com os tipos de substâncias e de suas características sociodemográficas. No que diz respeito ao perfil sociodemográfico, nos participantes deste estudo houve prevalência de adultos jovens, em sua maioria homens, solteiros, com nível socioeconômico baixo e escolaridade baixa. Tais características vão ao encontro com demais estudos sobre o perfil de usuários de drogas do Brasil (Bastos \& Bertoni, 2014; Duailibi et al., 2008; Almeida, Anjos, Vianna, \& Pequeno, 2014). Identificar o perfil sociodemográfico dos usuários de drogas facilita entender o contexto social onde os mesmos estão inseridos para pensarmos alternativas mais promissoras de tratamento.

Em relação ao uso de droga de acordo com o sexo, as mulheres estiveram associadas ao consumo de crack, enquanto os homens não apresentaram tal associação. De acordo com um estudo americano, apesar de 
Tabela 2

Média, Desvio Padrão, Coeficientes de Confiabilidade e Correlação Item-Total para as Pontuações dos Fatores DASS

\begin{tabular}{|c|c|c|c|c|c|}
\hline \multirow{2}{*}{$\begin{array}{c}\text { DASS - fatores } \\
\text { e itens }\end{array}$} & \multicolumn{2}{|c|}{ Medidas descritivas } & \multicolumn{3}{|c|}{ Análise de confiabilidade } \\
\hline & Média & $\begin{array}{l}\text { Desvio } \\
\text { padrão }\end{array}$ & $\begin{array}{r}\text { Correlação } \\
\text { item total }\end{array}$ & $\begin{array}{l}\text { Alfa de Cronbach se } \\
\text { item deletado }\end{array}$ & $\begin{array}{c}\text { Alfa de } \\
\text { Cronbach }\end{array}$ \\
\hline Depressão & & & & & 0,867 \\
\hline 3 & 0,73 & 1,09 & 0,423 & 0,877 & \\
\hline 5 & 0,76 & 0,96 & 0,465 & 0,869 & \\
\hline 10 & 0,67 & 0,93 & 0,585 & 0,855 & \\
\hline 13 & 1,04 & 1,10 & 0,750 & 0,832 & \\
\hline 16 & 0,72 & 1,04 & 0,742 & 0,834 & \\
\hline 17 & 0,84 & 1,13 & 0,769 & 0,829 & \\
\hline 21 & 0,86 & 1,20 & 0,757 & 0,830 & \\
\hline Ansiedade & & & & & 0,859 \\
\hline 2 & 0,74 & 1,06 & 0,376 & 0,875 & \\
\hline 4 & 0,60 & 0,98 & 0,547 & 0,850 & \\
\hline 7 & 0,43 & 0,83 & 0,618 & 0,842 & \\
\hline 9 & 0,71 & 1,09 & 0,706 & 0,827 & \\
\hline 15 & 0,62 & 0,98 & 0,803 & 0,815 & \\
\hline 19 & 0,88 & 1,08 & 0,670 & 0,833 & \\
\hline 20 & 0,83 & 1,09 & 0,703 & 0,828 & \\
\hline Estresse & & & & & 0,765 \\
\hline 1 & 1,12 & 1,09 & 0,499 & 0,264 & \\
\hline 6 & 1,04 & 1,09 & 0,622 & 0,398 & \\
\hline 8 & 0,87 & 1,05 & 0,712 & 0,532 & \\
\hline 11 & 1,15 & 1,02 & 0,658 & 0,469 & \\
\hline 12 & 1,23 & 1,10 & 0,691 & 0,521 & \\
\hline 14 & 1,02 & 1,09 & 0,695 & 0,503 & \\
\hline 18 & 1,38 & 1,08 & 0,588 & 0,384 & \\
\hline
\end{tabular}

a população feminina ter menores chances de se envolver com o uso de drogas, essas usuárias tendem a ter um consumo em maior quantidade de crack do que as demais drogas, enquanto homens tendem a usar diferentes substâncias de forma concomitante (Palamar, Davies, Ompad, Cleland, \& Weitzman, 2015). Destaca-se ainda que os dados do Perfil Nacional sobre Usuários de Crack apontam que mulheres relatam consumir 21 pedras de crack no dia de maior consumo, enquanto que homens relatam 13 pedras no mesmo período (Bastos \& Bertoni, 2014). Dessa forma, a intensidade do uso também necessita ser considerada no tratamento, de acordo com as especificidades de homens e mulheres (Limberger, Schneider, \& Andretta, 2015).

Corroborando com a presente pesquisa, a presença de baixos níveis de sintomas depressivos e ansiosos também foi encontrada em um estudo brasileiro com 60 apenados de um presídio, no qual 80\% dos participantes eram usuários de diferentes tipos de drogas (Tavares, Scheffer, \& Almeida, 2012). No referido estudo, identificou-se $63 \%$ dos participantes apresentaram níveis leves de sintomas depressivos e 
Tabela 3

Classificação dos Participantes no DASS-21

\begin{tabular}{|c|c|c|c|}
\hline Subclasse & Classificação & Frequência & Porcentagem \\
\hline \multicolumn{4}{|l|}{ Depressão } \\
\hline & Normal & 92 & 54,8 \\
\hline & Médio & 20 & 11,9 \\
\hline & Moderado & 23 & 13,7 \\
\hline & Severo & 10 & 6,0 \\
\hline & Extremamente severo & 23 & 13,7 \\
\hline & Total & 168 & 100,0 \\
\hline \multicolumn{4}{|l|}{ Ansiedade } \\
\hline & Normal & 96 & 57,1 \\
\hline & Médio & 17 & 10,1 \\
\hline & Moderado & 15 & 8,9 \\
\hline & Severo & 10 & 6,0 \\
\hline & Extremamente severo & 30 & 17,9 \\
\hline & Total & 168 & 100,0 \\
\hline \multicolumn{4}{|l|}{ Estresse } \\
\hline & Normal & 97 & 57,7 \\
\hline & Médio & 16 & 9,5 \\
\hline & Moderado & 16 & 9,5 \\
\hline & Severo & 19 & 11,3 \\
\hline & Extremamente severo & 20 & 11,9 \\
\hline & Total & 168 & 100,0 \\
\hline
\end{tabular}

25\% de sintomas ansiosos de nível mínimo. Alem disso, no contexto do presídio, os baixos níveis de sintomas depressivos e ansiosos podem ser compreendidos pela diminuiçãodo contato e do consumo de drogas ocasionado pela prisão (Tavares et al., 2012). No presente estudo, os locais de tratamento de internação também diminuem o acesso ao uso de drogas o que pode justificar tais resultados.

No que diz respeito à relação encontrada entre participantes que não estavam trabalhando e que apresentaram níveis severos e extremamente severos de depressão, evidencia-se importância do trabalho na vida dos usuários de substâncias. Em um estudo qualitativo com 28 mulheres usuárias de drogas em tratamento no Canadá, o trabalho foi observado como uma necessidade aliada ao tratamento, contribuindo assim no sentimento de pertencimento à sociedade (Kruk \& Sandberg, 2013). Em um estudo brasileiro com 93 mulheres, o trabalho remunerado foi considerado fator de proteção, pois apresentou um risco cerca de cinco vezes menor de apresentar depressão (Polisseni et al., 2009).

Também em relação ao âmbito profissional, Melo, Oliveira e Ferreira (2006) realizaram um estudo com 109 usuários de tabaco e identificaram que destes, 60,6\% não possuíam vínculo empregatício e, quando avaliados em relação aos índices de ansiedade e depressão, 32,3\% indicaram índices de ansiedade leve e 29,3\% índices moderados de depressão. Giatti e Barreto (2011) estudaram uma população de 62.471 pessoas e os resultados indicaram a associação entre ter um trabalho sem proteção social ou estar desempregado à maior prevalência de tabagismo entre homens e mulheres. Nessa perspectiva, destaca-se a importância de abordar aspectos da vida profissional em locais de tratamento para usuários de drogas. Compreende-se que estar empregado se relaciona a menores níveis de depressão, ansiedade e estresses, e que tais sintomatologias altas podem prejudicar no processo de recuperação dos usuários de substâncias. 
Tabela 4

Associações entre Depressão, Ansiedade e Estresse e Uso de Tabaco

\begin{tabular}{|c|c|c|c|c|}
\hline \multirow[b]{2}{*}{ Depressão } & \multirow[t]{2}{*}{ Classificação } & \multicolumn{2}{|c|}{ Usuário de tabaco? } & \multirow{2}{*}{$\frac{\mathrm{p}}{0,009 * *}$} \\
\hline & & $\operatorname{Sim}$ & Não & \\
\hline & Normal & 30 & 62 & \\
\hline & Médio & 7 & 13 & \\
\hline & Moderado & 14 & 9 & \\
\hline & Severo & 7 & 3 & \\
\hline & Extremamente Severo & 14 & 9 & \\
\hline \multirow[t]{6}{*}{ Ansiedade } & & & & $0,006^{* *}$ \\
\hline & Normal & 32 & 64 & \\
\hline & Médio & 7 & 10 & \\
\hline & Moderado & 6 & 9 & \\
\hline & Severo & 8 & 2 & \\
\hline & Extremamente Severo & 19 & 11 & \\
\hline \multirow[t]{6}{*}{ Estresse } & & & & $0,006 * *$ \\
\hline & Normal & 32 & 64 & \\
\hline & Médio & 7 & 10 & \\
\hline & Moderado & 6 & 9 & \\
\hline & Severo & 8 & 2 & \\
\hline & Extremamente Severo & 19 & 11 & \\
\hline \multirow[t]{4}{*}{ Depressão } & & & & $0,001^{* *}$ \\
\hline & Normal e Médio & 37 & 75 & \\
\hline & Moderado & 14 & 9 & \\
\hline & Severo e Extremamente Severo & 21 & 12 & \\
\hline \multirow[t]{4}{*}{ Ansiedade } & & & & $0,001 * *$ \\
\hline & Normal e Médio & 39 & 74 & \\
\hline & Moderado & 6 & 9 & \\
\hline & Severo e Extremamente Severo & 27 & 13 & \\
\hline \multirow[t]{4}{*}{ Estresse } & & & & $0,0001 * *$ \\
\hline & Normal e Médio & 34 & 79 & \\
\hline & Moderado & 12 & 4 & \\
\hline & Severo e Extremamente Severo & 26 & 13 & \\
\hline
\end{tabular}

Nota. Variáveis analisadas pelo Teste de Qui-Quadrado ${ }^{1}$ Agrupamento conforme o instrumento ${ }^{2}$ Agrupamento em três subgrupos. ${ }^{* *} p$ significativo ao nível de 0,01 .

A única substância que revelou ter relação com sintomas de depressão, ansiedade e estresse, neste estudo, foi o tabaco, corroborado por outras pesquisas já realizadas. Em relação a depressão e o tabaco, também foram identificados resultados referentes a diferentes populações. Alguns estudos destacam a presença da correlação positiva com o uso de tabaco e depressão em pacientes cardíacos (Stafford, Berk, \& Jackson, 2013), a associação entre aumento de risco de depressão na vida adulta em crianças com exposição a fumaça do cigarro (Elmasry, Goodwin, Terry, \& Tehranifar, 2014) e sintomas depressivos em fumantes com alta dependência em programa de cessão de tabagismo (Karen, Oliveira, Pádua, Vieira, \& Martinez, 2012). 
Apesar do número de usuários de tabaco que apresentaram depressão severa ou extremamente severa não ter sido relativamente alto na amostra, destaca-se os riscos que fumantes de cigarro possuem para o desenvolvimento de sintomatologia depressiva, envolvendo a diminuição da qualidade de vida dos mesmos. Essa perspectiva vai ao encontro da identificação do aumento significativo de risco de desenvolvimento de depressão naqueles que fumavam cigarro em um estudo dinamarquês que avaliou 18.146 participantes adultos, acima de 20 anos, por um período de até 26 anos (Flensborg-Madsen et al., 2011). Em outro estudo realizado com usuários de tabaco, identificou-se que os escores de ansiedade e depressão eram menores nos pacientes que haviam aderido ao tratamento quando comparado aos que não aderiam (Melo et al., 2006). A partir de tais constatações, enfatiza-se a importância de que os locais de internação para usuários de drogas incluam aspectos referentes a motivação para o tratamento, a fim de viabilizar maior adesão do paciente, e também aspectos relacionados a cessação do tabagismo.

O uso de tabaco também foi relacionado significativamente ao estresse na amostra estudada. O uso do cigarro tem sido observado em diferentes pesquisas como forma de lidar com situações estressantes, buscando na substância um alívio de tensão momentânea (Lopes, Cunha, Zibetti, \& Bizarro, 2014; McGee, Williams, Nada-Raja, \& Olsson, 2013). Estar em tratamento se revela uma situação geradora de estresse, e o cigarro pode ser uma das formas encontradas para lidar com os fatores estressantes, sendo um manejo disfuncional. Destaca-se a necessidade de envolver no tratamento para transtorno por uso de substâncias, intervenções que visem manejos de enfrentamento adaptativos ao estresse, sem que estes precisem utilizar o cigarro para isso.

O instrumento DASS-21 se mostrou adequado para avaliar a sintomatologia de depressão, ansiedade e estresse na amostra estudada de indivíduos em tratamento por transtorno por uso de substância, apresentando uma boa confiabilidade. Percebe-se que tal instrumento já tem sido utilizado em outras populações clínicas (Akin \& Iskender, 2011; Hopley \& Nick, 2010), bem como para a avaliação de comportamentos aditivos. Entretanto, pesquisas com o DASS-21 relacionadas a transtorno por uso de substâncias foram escassas. Uma pesquisa realizada na Turquia encontrou associação positiva entre tais sintomas e dependência de internet em uma amostra de 300 universitários adultos jovens (Akin \& Iskender, 2011). Tais sintomas também foram identificados como preditores em jogadores compulsivos de poker online no Canadá (Hopley \& Nick, 2010). Sobre o uso de substância, um estudo em Portugal utilizou-se o DASS-21 na perspectiva de avaliar de maneira geral os sintomas de depressão, ansiedade e estresse como possíveis moderadores na intenção de parar de fumar (Afonso \& Alves, 2013). Os resultados apontaram que maiores sintomas podem ser fatores de manutenção do uso de tabaco, causando a falsa percepção de qualidade de vida com seu consumo. A partir dos resultados encontrados na amostra da presente pesquisa e, também, dos estudos encontrados, percebe-se a relevância de utilizar o DASS-21 apresentando-se como um instrumento adequado para avaliar tais sintomatologias em populações com transtornos aditivos, a fim de conhecer para intervir, de forma adequada, em relação a depressão, ansiedade e estresse.

As diferenças entre homens e mulheres foram apontadas em relação ao uso do crack, onde mulheres utilizavam o crack de maneira exclusiva, enquanto homens utilizavam as drogas de maneira concomitantemente. Entretanto, tais dados são limitados ao contexto onde foram realizadas as coletas de dados da presente pesquisa. Também se destaca como limitação do estudo o tamanho da amostra, não sendo passível de generalização dos dados para todas as populações usuários de drogas. Desta forma, sugere-se que os níveis de depressão, ansiedade e estresse sejam avaliados em outros contextos de tratamento, bem como em diferentes etapas do tratamento, enfatizando a importância de verificar as relações de tais sintomatologias com diferentes substâncias.

A avaliação dos níveis de ansiedade, depressão e estresse em usuários de drogas contemplou o objetivo proposto, identificando-se associações significativas entre o uso do tabaco e níveis de depressão, ansiedade e estresse, bem como não estar trabalhando e apresentar níveis severos e extremamente severos de depressão. Entretanto, cabe destacar que tais dados não permitem uma relação de causalidade entre as variáveis analisadas, sugerindo que futuros estudos possam identificar variáveis que predizem a depressão, ansiedade e estresse em usuários de drogas em tratamento.

Ressalta-se que o uso de tabaco muitas vezes não é abordado nos locais de tratamento, sendo o foco no álcool e nas drogas ilícitas. Dessa forma, o presente estudo alerta para os prejuízos que o uso de tabaco pode ter para a recuperação dos usuários de drogas, com maiores níveis de depressão, ansiedade e estresse que podem afetar negativamente no tratamento. Com 
isso, tais variáveis necessitam ser consideradas no tratamento do usuário de drogas, a fim de repensar a reinserção profissional e o manejo das emoções, diminuindo os fatores de risco para o retorno ao uso de drogas.

\section{Referências}

Associação Brasileira de Empresas de Pesquisa [ABEP]. (2015). Critérios de Classificaşão Econômica Brasil. Recuperado de http:/ / www.abep.org/criteriobrasil

Afonso, M. F. B., \& Alves, M. G. P. (2013). Morbidade psicológica como moderador da intenção para deixar de fumar: um estudo com fumantes e ex-fumantes. Jornal Brasileiro de Pneumologia, 39(4), 461-468. doi: 10.1590/S1806-37132013000400010

Akin, A., \& Iskender, M. (2011). Internet addiction and depression, anxiety and stress. International online journal of educational sciences, 3(1), 138-148. Recuperado de http://www.iojes.net/userfiles/Article/ IOJES_436.pdf

Almeida, R. A., Anjos, U. U., Vianna, R. P. T., \& Pequeno, G. A. (2014). Perfil dos usuários de substâncias psicoativas de João Pessoa. Saúde Debate, 38(102), 526-538. doi: 10.5935/0103-1104.20140049

American Psychiatric Association. (2002). Manual Diagnóstico e Estatístico de Transtornos Mentais. DSMIV-TR. Porto Alegre: Artmed.

American Psychiatric Association. (2014). Manual Diagnóstico e Estatístico de Transtornos Mentais. DSM-5. Porto Alegre: Artmed.

Amorim, P. (2000). Mini International Neuropsychiatric Interview (MINI): Validação de entrevista breve para diagnóstico de transtornos mentais. Revista Brasileira de Psiquiatria, 22(3), 106-115. doi: 10.1590/ S1516-44462000000300003

Apóstolo, J. L. A., Mendes, A. D., \& Azeredo, Z. A. (2006). Adaptação para a língua portuguesa da Depression, Anxiety and Stress Scale (DASS). Revista Latinoamericana de Enfermagem, 14(6). doi: 10.1590/ S0104-11692006000600006

Argimon, I. I., Terroso, L. B., Farina, M., Moraes, A. A., Lopes, R. M. F., \& Querotti, K. L. M. (2013). A intensidade da depressão e a internação de alcoolistas. Aletheia, 40, 102-110. Recuperado de http:// pepsic.bvsalud.org/pdf/aletheia/n40/n40a09.pdf
Bastos, F. I. P. M., \& Bertoni, N. (2014). Pesquisa Nacional sobre o uso de crack: Quem são os usuários de crack e/ou similares do Brasil? Quantos são nas capitais brasileiras? Recuperado de https:// www.icict.fiocruz.br/sites/www.icict.fiocruz.br/ files $/$ Pesquisa $\% 20$ Nacional $\% 20$ sobre $\% 20$ o $\% 20$ Uso $\% 20$ de $\% 20$ Crack.pdf

Beck, A. T., \& Alford, B. A. (2011). Depressão: Causas e tratamento. São Paulo: Artmed.

Boschloo, L., Vogelzangs, N., Smit, J. H., van den Brink, W., Veltman, D. J., Beekman, A. T., \& Penninx, B. W. (2011). Comorbidity and risk indicators for alcohol use disorders among persons with anxiety and/or depressive disorders: Findings from the Netherlands Study of Depression and Anxiety (NESDA). Journal of Affective Disorders, 131(1), 233-242. doi: 10.1016/j. jad.2010.12.014

Castro, M. M., Quarantini, L. C., Daltro, C., Pires-Caldas, M., Koenen, K. C., Kraychete, D. C., \& de Oliveira, I. R. (2011). Comorbidade de sintomas ansiosos e depressivos em pacientes com dor crônica e o impacto sobre a qualidade de vida. Revista de Psiquiatria Clínica, 38(4), 126-9. doi:10.1590/ S0101-60832011000400002

Conner, K. R., Pinquart, M., \& Holbrook, A. P. (2008). Meta-analysis of depression and substance use and impairment among cocaine users. Drug and Alcohol Dependence, 98(1), 13-23. doi: 10.1016/j. drugalcdep.2008.05.005

Cunha, J. A. (1993). Psicodiagnóstico-R. 4. ed. Porto Alegre: Artes Médicas.

Diehl, A., Cordeiro, D. C., \& Laranjeira, R. (2011). Dependência química: Prevenção, tratamento e políticas públicas. Porto Alegre: Artmed.

Duailibi, L. B., Ribeiro, M., \& Laranjeira, R. (2008). Profile of cocaine and crack users in Brazil. Caderno de Saúde Pública, 24(4), 545-557. doi: 10.1590/ S0102-311X2008001600007

Elmasry, H., Goodwin, R. D., Terry, M. B., \& Tehranifar, P. (2014). Early life exposure to cigarette smoke and depressive symptoms among women in midlife. Nicotine \& Tobacco Research, 16(10), 1298306. doi: $10.1093 / \mathrm{ntr} / \mathrm{ntu} 070$

Enoch, M. (2003). Pharmacogenomics of alchol respnse and addiction. American Journal 
of Pharmacogenomics, 3(4), 217-232. doi: 10.2165/00129785-200303040-00001

Federação Brasileira de Comunidades Terapêuticas [FEBRACT]. (2016). Filidadas. Recuperado de http://www.febract.org.br/?navega=filiadas

Feldens, A. C. M., Silva, J. G. D., \& Oliveira, M. D. S. (2011). Avaliação das funções executivas em alcoolistas. Cadernos de Saúde Coletiva, 19, 164-171. Recuperado de http://www.cadernos.iesc.ufrj. br/cadernos/images/csc/2011_2/artigos/csc_ v19n2_164-171.pdf

Flensborg-Madsen, T., von Scholten, M. B., Flachs, E. M., Mortensen, E. L., Prescott, E., \& Tolstrup, J. S. (2011). Tobacco smoking as a risk factor for depression. A 26-year population-based follow-up study. Journal of Psychiatric Research, 45(2), 143-149. doi: 10.1016/j.jpsychires.2010.06.006

Fox, T. P., Oliver, G., \& Ellis, S. M. (2013). The destructive capacity of drug abuse: An overview exploring the harmful potential of drug abuse both to the individual and to society. ISRN Addiction. Article ID 450348. doi: 10.1155/2013/450348

Giatti, L., Barreto, S. M. (2011). Tabagismo, situação no mercado de trabalho e gênero: Análise da PNAD 2008. Cadernos de Saúde Pública, 27(6), 1132-1142. doi: 10.1590/S0102-311X2011000600010

Goeders, N. E. (2003). The impact of stress on addiction. Eur Neuropsuchopharmacol, 13(6), 435-41. doi: 10.1016/j.euroneuro.2003.08.004

Hess, A. R. B., Almeida, R. M. M. D., \& Moraes, A. L. (2012). Comorbidades psiquiátricas em dependentes químicos em abstinência em ambiente protegido. Estudos de Psicologia, 17(1), 171-178. doi: 10.1590/S1413-294X2012000100021

Hofmann, S. G., Richey, J. A., Kashdan, T. B., \& McKnight, P. E. (2009). Anxiety disorders moderate the association between externalizing problems and substance use disorders: Data from the National Comorbidity Survey-Revised. Journal of Anxiety Disorders, 23(4), 529-534. doi: 10.1016/j. janxdis.2008.10.011

Hopley, A. A., \& Nicki, R. M. (2010). Predictive factors of excessive online poker playing. Cyberpsychology, Behavior, and Social Networking, 13(4), 379-385. doi: 10.1089/cyber.2009.0223
Karen, S. K., Oliveira, M. L., Pádua, A. I., Vieira, F., \& Martinez, J. A. (2012). Características clínicas de fumantes atendidos em um centro de referência na cessação do tabagismo. Medicina (Ribeirão Preto. Online), 45(3), 337-342. doi: 10.11606/issn.2176-7262. v45i3p337-342

Kruk, E., \& Sandberg, K. A (2013). A home for body and soul: Substance using women in recovery. Harm Reduction Journal, 10(39). doi: 10.1186/1477-7517-10-39.

Laranjeira, R., Madruga, C. S., Pinsky, I., Caetano, R., Ribeiro, M., \& Mitsuhiro, S. II Levantamento Nacional de Álcool e Drogas (LENAD) - Uso de cocaína e crack no Brasil. (2012). São Paulo, SP: INPAD. Recuperado de http://inpad.org.br/lenad/ cocaina-e-crack/resultados-preliminares/

Limberger, J., Schneider, J., \& Andretta, I. (2015). Especificidades do tratamento de mulheres usuárias de crack: interface com direitos humanos. Psicologia em Pesquisa, 9(2), 139-147. doi: 10.5327/ Z1982-1247201500020004

Lopes, F., Cunha, S. M. D., Zibetti, M., \& Bizarro, L. (2014). Padrão de consumo e expectativas em relação ao cigarro entre universitários. Psicologia, Saúde \& Doenças, 15(2), 439-453. doi: 10.15309/14psd150209

Marcon, S. R., Xavier, J. S., Barcelon, A. A., Espinosa, M. M., \& Barbosa, D. A. (2014). Correlação entre sintomas depressivos e qualidade de vida de usuários de substâncias psicoativas. Revista da Escola de Enfermagem da USP, 48(4), 663-669. doi: 10.1590/ S0080-623420140000400013

Marmorstein, N. R. (2012). Anxiety disorders and substance use disorders: Different associations by anxiety disorder. Journal of Anxiety Disorders, 26(1), 88-94. doi: 10.1016/j.janxdis.2011.09.005

McGee, R., Williams, S., Nada-Raja, S., \& Olsson, C. A. (2013). Tobacco smoking in adolescence predicts maladaptive coping styles in adulthood. Nicotine \& Tobacco Research, ntt081. doi: 10.1093/ntr/ntt081

Melo, W. V., Oliveira, M. S., \& Ferreira, E. A. (2006). Estágios motivacionais, sintomas de ansiedade e depressão no tratameto do tabagismo. Interação em Psicologia, 10(1), 91-99. doi: 10.5380/psi.v10i1.5769

Munaretti, C. L., \& Terra, M. B. (2007). Transtornos de ansiedade: Um estudo de prevalência e 
comorbidade com tabagismo em um ambulatório de psiquiatria. Jornal Brasileiro de Psiquiatria, 56(2), 108-115. doi: 10.1590/S0047-20852007000200006

Palamar, J. J., Davies, S., Ompad, D. C., Cleland, C. M., \& Weitzman, M. (2015). Powder cocaine and crack use in the United States: An examination of risk for arrest and socioeconomic disparities in use. Drug and Alcohol Dependence, 149, 108-116. doi: 10.3109/00952990.2012.703735

Polisseni, A. F., Araújo, D. A. C., Polisseni, F., Mourão Junior, C. A., Polisseni, J., Fernandes, E. S., \& Guerra, M. O. (2009). Depressão e ansiedade em mulheres climatéricas: Fatores associados. Revista Brasileira de Ginecologia e Obstetrícia, 31(1), 28-34. doi: 10.1590/S0100-72032009000100006

Saatcioglu, O., Yapici, A., \& Cakmak, D. (2008). Quality of life, depression and anxiety in alcohol dependence. Drug and Alcohol Review, 27(1), 83-90. doi: 10.1080/09595230701711140

Sampieri, R. H., Collado, C. F., \& Lucio, M. P. B. (2013). Metodologia de Pesquisa. Porto Alegre: Penso.

Scheffer, M., Passa, G. G., \& Almeida, R.M. (2010). Dependência de álcool, cocaína e crack e transtornos psiquiátricos. Psicologia: Teoria e Pesquisa, 26(3), 533541. doi: 10.1590/S0102-37722010000300016

Sinha, R. (2009). Modeling stress and drug craving in the laboratory: Implications for addiction treatment development. Addiction Biology, 14(1), 84-98. doi: 10.1111/j.1369-1600.2008.00134.x

Stafford, L., Berk, M., \& Jackson, H. J. (2013). Tobacco smoking predicts depression and poorer quality of life in heart disease. BMC Cardiovascular Disorders, 13(1), 35. doi: 10.1186/1471-2261-13-35

Staiger, P. K., Thomas, A. C., Ricciardelli, L. A., \& McCabe, M. P. (2011). Identifying depression and anxiety disorders in people presenting for substance use treatment. Medical Journal of Australia, 195(3). Recuperado de https://www.mja. com.au/system/files/issues/195_03_010811/ sta10926_fm.pdf

Tavares, G. P., Scheffer, M., \& Almeida, R. M. M. (2012). Drogas, violência e aspectos emocionais em apenados. Psicologia: Reflexão e Crítica, 25(1), 89-95. doi: 10.1590/S0102-79722012000100011

United Nations Office on Drugs and Crime [UNODC], World Drug Report 2015 (United Nations publication, Sales No. E.15.XI.6). Recuperado de https://www.unodc.org/documents/wdr2015/ World_Drug_Report_2015.pdf

Wechsler, D. (1997). Weschsler Adult Intelligence Scale-III. San Antonio: The Psychological Corporation.

Recebido em: 25/01/2016

Reformulado em: 16/11/2016 Aprovado em: 10/04/2017 
Sobre as autoras:

Ilana Andretta é psicóloga Clínica, mestra em Psicologia (PUCRS), doutora em Psicologia (PUCRS) e professora no Programa de Pós-Graduação em Psicologia Clínica da Universidade do Vale do Rio dos Sinos (UNISINOS), onde coordena o Grupo de Pesquisa ICCep - Intervenções Cognitivo Comportamentais: Ensino e Pesquisa. Desenvolve ainda pesquisas sobre TICs, Habilidades Sociais, Treinamento em Habilidades Sociais e Transtornos Relacionados ao Uso de Substâncias.

E-mail:ilana.andretta@gmail.com

Jéssica Limberger é psicóloga clínica, mestra em Psicologia Clínica (UNISINOS), doutoranda em Psicologia Clínica na Universidade do Vale do Rio dos Sinos (UNISINOS), bolsista CAPES/PROSUC e integrante do Grupo de Pesquisa ICCep - Intervenções Cognitivo Comportamentais: Ensino e Pesquisa (UNISINOS). Desenvolve ainda pesquisas sobre habilidades sociais, Treinamento em Habilidades Sociais e Transtornos Relacionados ao Uso de Substâncias.

ORCID: https://orcid.org/0000-0002-5205-7143

E-mail: jessica.limberger.psi@gmail.com

Jaluza Aimèe Schneider é psicóloga clínica, mestre em Psicologia Clínica (2015) pela Universidade do Vale do Rio dos Sinos, doutoranda em Psicologia Clínica pela Pontifícia Universidade Católica do Rio Grande do Sul (PUCRS) e realiza atendimentos psicológicos clínicos na abordagem cognitivo-comportamental.

ORCID: https://orcid.org/0000-0001-7899-0786

E-mail: jaluzas@gmail.com

Luana Thereza Nesi de Mello é graduanda em Psicologia pela Universidade do Vale do Rio dos Sinos (UNISINOS), bolsista PROBIC/FAPERGS no Grupo de Pesquisa Intervenções Cognitivo Comportamentais: Ensino e Pesquisa (ICCep) e possui experiência como aluna de iniciação científica em pesquisas com enfoque em Habilidades Sociais, Transtornos Relacionados ao Uso de Substâncias, Adolescentes, Mindfulness e TICs.

ORCID: https://orcid.org/0000-0003-4139-6681

E-mail: luana.nesi@gmail.com

Contato com as autoras:

Ilana Andretta

Universidade do Vale do Rio dos Sinos

Av. Unisinos, 950. Escola de Saúde. Programa de Pós-Graduação em Psicologia Clínica. Bairro Cristo Rei

São Leopoldo-RS, Brasil

CEP: 93020-190

ORCID: https://orcid.org/

0000-0002-5537-5120 